\title{
Young atmosphere in the eternal city: the Third Educational Colorectal Meeting
}

\author{
Federica Cadeddu • Giovanni Milito
}

Published online: 2 February 2011

(C) Springer-Verlag 2011

Building on the success of the two previous meetings, the Third Educational Colorectal Meeting took place in Rome on 13th-14th December 2010, organized by SICCR and chaired by F. La Torre and G. Milito.

A young atmosphere has characterized the Third Educational Colorectal Meeting: the congress was focused on the podium presentations performed by young surgeons and followed by interesting and useful face to face with senior referees who had previously received the abstracts and focused the attention on the main discussion points.

The focus of the meeting was on colorectal surgical emergencies: colorectal bleeding, bowel obstruction and perforation, pain, anastomotic leakages, perineal injuries. The program committee selected 30 papers for podium presentation and 15 for poster discussion.

In particular, several key points of colorectal emergencies were highlighted and debated; such as the increasing role of laparoscopy to treat colorectal emergencies and to manage acute small bowel obstructions; the surgical versus endoscopic palliation for bowel obstruction; the role of diverting stoma in emergency colorectal surgery with regard to indication, morbidity, outcomes. Besides, the topic of colorectal anastomotic leak was also addressed with a focus on incidence, risk factors, and treatment either surgical or endoscopic.

Furthermore, the meeting was improved by the participation of outstanding speakers, including R. J. Nicholls presenting a lecture on acute colitis, S. D. Wexner presenting a lecture on post-operative treatment of anastomotic leakage, M. Behro who discussed the role of TME in the surgical management of rectal cancer, and finally C. Mavrantonis who focused his presentation on laparoscopy in the management of acute diverticulitis. Finally, an outstanding face to face on Crohn Disease took place by M. Behro, R. J. Nicholls, G. Riegler, and S. D. Wexner with an interesting discussion based on different case reports.

Last but not least, the Meeting was enriched by the wellorganized social program including the visit to the Van Gogh exhibition and the dinner in a special location: Piazza Venezia.

Above all, the Meeting was mainly improved by all the speakers who participated in interesting and lively sessions with useful discussions, by the high quality of the presentations and by the warmth of atmosphere, and this successful outcome makes us look forward to the next Educational Meeting hopefully in the near future.
F. Cadeddu · G. Milito ( $\square)$

University Hospital Tor Vergata, Rome, Italy

e-mail: giovanni.milito@virgilio.it 